our calculation (the neglect of lattice dynamics and the use of perfect crystalline lattices). Both plasma models may be compared to the Monte Carlo simulations of Hubbard and DeWitt (9) (at 10.5 Mbar), who used the Lindhard dielectric function to screen the bare Coulomb interaction between the ions (dashed curve in Fig. 3 labeled Lindhard). The Lindhard results are very close to both the OCP-LM and ion-sphere curves but very far from our calculations.

Our phase-separation temperatures were calculated for a pressure of $10.5 \mathrm{Mbar}$ but are virtually independent of pressure in the range from 5 to $20 \mathrm{Mbar}$. However, at low pressures hydrogen is a molecular insulator and becomes a molecular metal near $2 \mathrm{Mbar}$ (5). Van den Bergh and Schouten (17), using pair potentials fit to low-pressure experiments, found that for pressures up to 1 Mbar helium will be completely miscible in insulating, molecular hydrogen for temperatures above approximately $2500 \mathrm{~K}$. The miscibility of helium in metallic, molecular hydrogen is unknown. Our calculations do not apply directly to this molecular phase, but in analogy with the metallization of pure helium we expect that the precise pressure at which molecular hydrogen metallizes will be unimportant with regard to the miscibility. Rather, we expect that the phase-separation temperatures will increase monotonically from van den Bergh and Schouten's values at $1 \mathrm{Mbar}$ to the values we calculate at sufficiently high pressure, where hydrogen is an atomic (rather than molecular) metal. Additional TE calculations for alloys containing molecular hydrogen are needed in order to accurately determine the behavior of the miscibility gap below 5 Mbar.

The primary conclusion obtained from the results of our TE calculations is that it is crucial to treat the electronic structure accurately in order to obtain the correct thermodynamics for hydrogen-helium mixtures at megabar pressures. The electronic energy makes a large contribution to the phaseseparation temperature. This temperature could be small only if we have severely underestimated the thermal contribution. Barring this circumstance, our phase-separation temperature of $15,000 \pm 3,000 \mathrm{~K}$ for a $7 \%$ helium mixture confirms that the fluid interior of Saturn has at least partially phaseseparated, because the maximum temperature in the fluid is estimated to be only $10,000 \mathrm{~K}$. The estimated temperatures in the fluid interior of Jupiter range from $10,000 \mathrm{~K}$ near the surface to $20,000 \mathrm{~K}$ at the central core (1). Thus our calculation predicts that phase separation has also begun in Jupiter. In view of this prediction, the fact that the currently successful evolutionary models of Jupiter do not need to invoke phase separation may indicate a failure of these models. Alternatively, phase separation may have occurred too late in the evolution of Jupiter to provide a significant internal energy source up to the present time. In either case, new evolutionary calculations are needed to resolve this dilemma and to confirm that the inclusion of phase separation leads to a consistent model of Saturn.

\section{REFERENCES AND NOTES}

1. A. S. Grossman, J. B. Pollack, R. T. Reynolds, A. L. Summers, H. C. Graboske, Icarus 42, 358 (1980); D. J. Stevenson, Annu. Rev. Earth Planet. Sci. 10, 257 (1982).

2. D. J. Stevenson and E. E. Salpeter, Astrophys. J. Suppl. Ser. 35, 221 (1977); ibid., p. 239.

3. R. A. Hanel, B. J. Conrath, V. G. Kunde, J. C Pearl, J. A. Pirraglia, Icarus 53, 262 (1983)

4. R. Smoluchowski, Nature 215, 691 (1967); E. E. Salpeter, Astrophys. J. 181, L83 (1973).

5. T. W. Barbee III, A. Garcia, M. L. Cohen, J. L. Martins, Phys. Rev. Lett. 62, 1150 (1989).

6. D. A. Young, A. K. McMahan, M. Ross, Phys. Rev. B 24, 5119 (1981).
7. D. J. Stevenson, ibid. 12, 3999 (1975)

8. E. L. Pollock and B. J. Alder, Phys. Rev. A 15, 1263 (1977); J. P. Hansen, G. M. Torrie, P. Vieillefosse, ibid. 16, 2153 (1977).

9. W. B. Hubbard and H. E. DeWitt, Astrophys. J. 290, 388 (1985).

10. J. J. MacFarlane, ibid. 280, 339 (1984).

11. See, for example, R. $O$. Jones and $O$. Gunnarsson, Rev. Mod. Phys. 61, 689 (1989).

12. See, for example, J. DeVreese Ed., Electronic Structure, Dynamics, and Quantum Structural Properties of Condensed Matter (Plenum, New York, 1985).

13. J. E. Klepeis, K. J. Schafer, T. W. Barbee III, M. Ross, Phys. Rev. B, in press.

14. B. Brami, J. P. Hansen, F. Joly, Physica A 95, 505 (1979); S. Ichimaru, H. Iyetomi, S. Ogata, Astrophys. J. 334, L17 (1988).

15. G. S. Stringfellow, H. E. DeWitt, W. L. Slattery, Phys. Rev. A 41, 1105 (1990)

16. E. E. Salpeter, Aust. J. Phys. 7, 373 (1954).

17. L. C. van den Bergh and J. A. Schouten, J. Chem. Phys. 89, 2336 (1988)

18. We are grateful to $M$. van Schilfgaarde for providing the linear muffin-tin orbital (LMTO) computer codes used in the calculations. We also acknowledge helpful discussions with A. K. McMahan. This work was performed under the auspices of the U.S. Department of Energy by the Lawrence Livermore National Laboratory under contract W-7405-ENG-48.

8 July 1991; accepted 22 August 1991

\title{
Superconductivity in the Fullerenes
}

\section{M. VARMA, J. ZaAnen, K. Raghavachari}

Intramolecular vibrations strongly scatter electrons near the Fermi-surface in doped fullerenes. A simple expression for the electron-phonon coupling parameters for this case is derived and evaluated by quantum-chemical calculations. The observed superconducting transition temperatures and their variation with lattice constants can be understood on this basis. To test the ideas and calculations presented here, we predict that high frequency $H_{g}$ modes acquire a width of about $20 \%$ of their frequency in superconductive fullerenes, and soften by about $5 \%$ compared to the insulating fullerenes.

$\mathrm{T}$ HE EXCITING DISCOVERY OF SUPERconductivity in metallic fullerenes (1) leads us to inquire whether the classic mechanism for superconductivity, namely, effective electron-electron attraction via the interaction of electrons with vibrations of the ions, is applicable here as well. Associated with this is the question of whether the direct electron-electron repulsion in Fullerenes can suppress conventional singlet pairing. In this paper we exploit the special nature of cluster compounds to derive a particularly simple expression for electronvibrational coupling from which parameters of the superconducting state of fullerenes are easily calculated. Further, we present arguments why the effective repulsions in fullerenes are no different than in conventional metals.

The lattice vibrations couple to the electronic states of metallic fullerenes in two

AT\&T Bell Laboratories, Murray Hill, NJ 07974. ways: by causing fluctuations in the hopping rate of electrons from one molecule to the other and by causing fluctuations in the electronic structure of a single molecule. The covalent interactions that split the molecular states, which form the bands in the metallic state, are over an order of magnitude larger than the inter-molecular covalency. This is reflected in the intra-molecular splitting $W_{\text {intra }} \simeq 20 \mathrm{eV}(2,3)$ compared to the width of the $t_{1 u}$ bands which is $W_{\text {inter }} \simeq 0.6 \mathrm{eV} \mathrm{(4,}$ $5)$. The electron-vibration coupling is known to be proportional to such covalent splittings (6). Therefore, in the problem of the fullerenes, one needs to consider only the intra-molecular vibration coupling. The same argument rationalizes why the electron-vibrational coupling may be much larger in doped fullerenes than in doped graphite. In the latter, the orbitals near the Fermienergy are $\pi$ bonded. The Fullerenes have a significantly larger relevant bandwidth, because of $\sigma$ admixture due to the non-planar local geometry, and therefore a stronger 


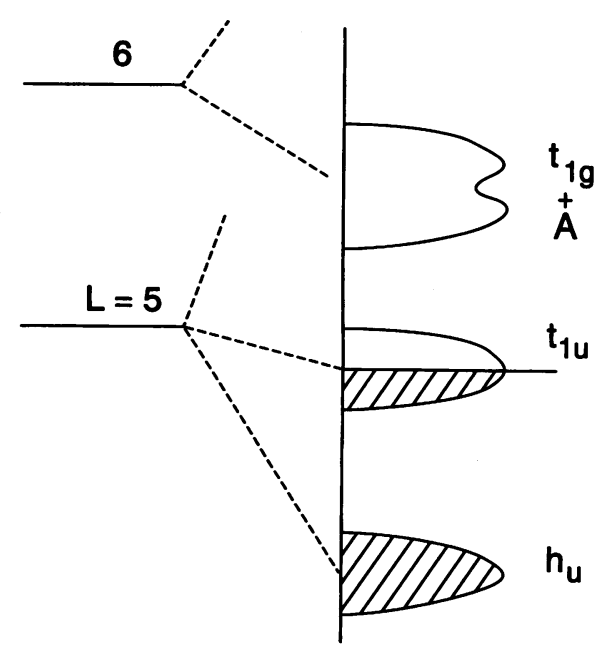

Fig. 1. Artists conception of the electronic structure of the fullerenes, as inferred from photoemission spectroscopies and electronic structure calculations. $A$ denotes the alkali $s$ level.

electron-phonon coupling (5).

Photoemission studies (7), in combination with electronic structure calculations $(2-4)$, have revealed a clear picture of the electronic structure of the fullerenes. The deviation from spherical symmetry of the icosahedra affects the $11 L=5$ spherical harmonic states (3), so that they split into a fivefold degenerate $h_{u}$ and two threefold degenerate $t_{1_{u}}$ and $t_{2_{u}}$ manifolds, respectively. As shown in Fig. 1 , the $h_{u}$ states lie $\sim 2$ $\mathrm{eV}$ below the $t_{1 u}$ states and are occupied. The $L=6$-derived $t_{1 g}$ states lie $\sim 1 \mathrm{eV}$ above the $t_{1 u}$ states, and the latter are occupied on doping. The unoccupied alkali $s$ states are roughly degenerate with the $t_{1 g}$ states (7). So one may confine attention simply to the threefold degenerate $t_{1 u}$ states. These transform as $x, y$, and $z$, and so their degeneracy is split by any quadrupolar deformation that makes the cartesian axes inequivalent. With respect to superconductivity these quadrupolar Jahn-Teller modes, having $H_{g}$ symmetry, are the only relevant ones. This follows from the fact that in icosahedral symmetry

$$
t_{1 u} \times t_{1 u}=A_{g}+T_{1 g}+H_{g}
$$

The $T_{1 g}$ mode is asymmetric so that it cannot couple, and the $A_{g}$ modes do not lift the degeneracy (although they change the local energy level).

The vibrational modes of the $\mathrm{C}_{60}$ molecules have been calculated. There are eight such $H_{g}$ modes, each of which is fivefold degenerate. So of the 174 vibrational modes of $\mathrm{C}_{60}, 40 \mathrm{can}$, in a static fashion (JahnTeller effect) or, more importantly, in a dynamic fashion (dynamic Jahn-Teller effect) affect the $t_{1 u}$ electronic states. Let us now consider the two limiting cases. The intramolecular processes can either lead to a pairing energy much larger than the intermolecular transfer integrals, or the reverse is true. The latter is found more appropriate for the fullerenes, but the former is interesting to discuss first because of its conceptual simplicity. Consider two molecules with average charge $\bar{n}$. The Hamiltonian for the coupled problem is a $3 \times 3$ matrix with elements

$$
H_{i j}=\bar{E} \delta_{i j}+\sum_{m, \mu} h_{i j}(m, \mu) \dot{Q_{m, \mu}}+H_{\text {vib }}
$$

where $i=1,2,3$ labels the degenerate states of $t_{1 u}$ symmetry, $Q_{m, \mu}$ are the normal coordinates of the $m$-th $H_{g}$ mode with degeneracy $\mu=1 \ldots 5 . H_{\text {vib }}$ is the Hamiltonian of the vibrational modes with frequencies $\omega_{m}$. This Jahn-Teller problem, involving a threefold degenerate electronic state interacting with a fivefold degenerate mode, has been worked out some time ago (8) and the coupling matrix is found to be

$$
\frac{1}{2} g_{m}\left(\begin{array}{cc}
Q_{m, 5}-\sqrt{3} Q_{m, 4}-\sqrt{3} Q_{m, 1}-\sqrt{3} Q_{m, 2} \\
-\sqrt{3} Q_{m, 1} Q_{m, 5}+\sqrt{3} Q_{m, 4}-\sqrt{3} Q_{m, 3} \\
-\sqrt{3} Q_{m, 2} & -\sqrt{3} Q_{m, 3}-2 Q_{m, 5}
\end{array}\right)
$$

where $g_{m}$ is the characteristic energy per unit displacement of the $m$-th mode, to be evaluated by microscopic calculations.

In the strong-coupling limit, the $t_{1 u}$ levels will split as indicated in Fig. 2 left for one phase of the vibration and Fig. 2 right for the other phase (8). Now if we calculate the energy reduction in this limit (neglecting the zeropoint motion) and deduce the effective electron-electron interaction through

$$
U_{\bar{n}}=E_{\bar{n}+1}+E_{\bar{n}-1}-2 E_{\bar{n}}
$$

where $E_{\bar{n}}$ is the ground state energy for

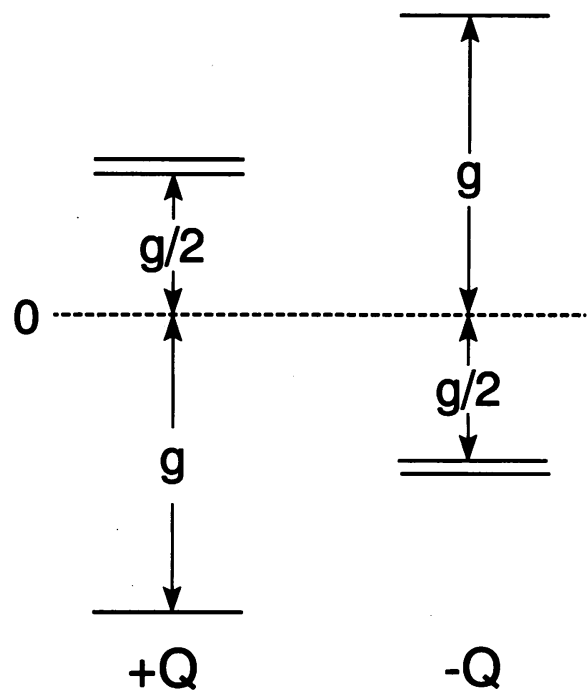

Fig. 2. The splitting pattern of the $t_{1 u}$ level by the $H_{g}$ distortion for the two different phases of the radial displacement $Q$.

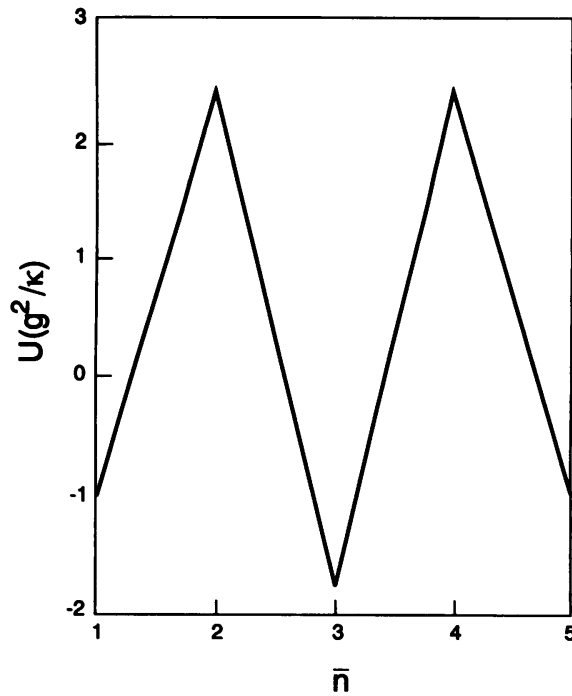

Fig. 3. The local electron-electron interaction $(U)$ as a function of particle number $(\bar{n})$, induced by the Jahn-Teller interactions ( $\kappa$ is the spring constant). A negative $U$ is found for an average occupation $(\overline{\bar{n}})$ of 3 electrons, and a positive $U$ for 2 or 4 electrons.

average charge $n$, we find the behavior as in Fig. 3 (9). The actual relevant electronelectron interaction parameter is in this limit, of course $\tilde{U}_{\bar{n}}=U_{\bar{n}}+U_{\mathrm{C}}$, where $U_{\mathrm{C}} \simeq$ $e^{2} / \varepsilon R \simeq 0.5$ to $1 \mathrm{eV}(\varepsilon \sim 4)$ comes from direct electron-electron interactions. $U_{\bar{n}}$ and $U_{\mathrm{C}}$ have in general different high frequency cutoffs.

This picture, which could yield an effective attractive interaction, would be a good starting point for further discussion if $-\widetilde{U}_{\bar{n}}$ $>W$, the conduction electron bandwidth (and the phonon frequency). One would then have local pairing (10) and a superconducting transition temperature of $\mathrm{O}\left(W^{2} /\right.$ $|\tilde{U}|)$, provided a static Jahn-Teller distortion with a possible phase difference from one molecule to the other, although a simple staggered charge density wave is frustrated in the fcc-lattice (11) were not to occur. For $\left|U_{\bar{n}}\right| \leq W$, as we find below, the above adiabatic picture is not valid-an electron runs away from a molecule before it develops pairing correlation with another electron on the same molecule. Then the physics we described above should be incorporated into the traditional way of considering electron-phonon scattering in metallic bands (6).

The 40 intramolecular $H_{g}$ modes are expected to be nearly dispersionless in the solid state. For this case, the electronphonon Hamiltonian is particularly simple, and may be written as

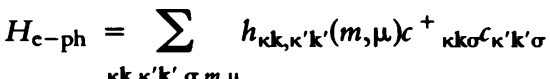

$$
\begin{aligned}
& \times\left(Q_{m \mu, \mathbf{k}-\mathbf{k}^{\prime}}+Q^{+}{ }_{m \mu,-\mathbf{k}}\right)
\end{aligned}
$$


Here the scattering matrix element

$$
h_{\mathrm{kk}, \kappa^{\prime} \mathbf{k}^{\prime}}(m, \mu)=\sum_{i j} A^{*}{ }_{k \mathbf{k}} h_{i j}(m \mu) A_{\mathbf{k}^{\prime} \mathbf{k}^{\prime} j}
$$

where $A_{\text {кki } i}$ are the elements of the linear transformation from molecular levels to band states- $k$ is the momentum and $k$ are the band indices.

For $s$-wave superconductivity, in weak to intermediate coupling, one is interested in time-reversed states near the Fermi-energy. So one may confine attention to intra-band pairing. For the present case, the dimensionless electron-phonon coupling constant $\lambda$ has a particularly simple form, which is easily derived from the general expressions $(12,13)$

$$
\begin{gathered}
\lambda=\sum_{m \mu} \frac{N(0)}{M \omega_{m}^{2}} I_{m \mu}^{2} \\
I_{m \mu}^{2}=N(0)^{-2} \sum_{k, k^{\prime}} \int \frac{d S_{\mathbf{k}, \kappa}}{v_{\mathbf{k}, \kappa}} \\
\times \int \frac{d S_{\mathbf{k}^{\prime}, \kappa^{\prime}}}{v_{\mathbf{k}^{\prime}, \kappa^{\prime}}}\left|h_{\mathbf{k}, \kappa^{\prime} \mathbf{k}^{\prime}}(m, \mu)\right|^{2}
\end{gathered}
$$

where the integrals are over the Fermisurface. Using the fact that the degeneracy of the $t_{1 u}$ levels is not lifted in a cubic environment (14) we find

$$
\lambda=\frac{5}{6} \sum_{m} \frac{N(0)}{M \omega_{m}^{2}} g_{m}^{2}
$$

We can now calculate the superconductive transition temperature $T_{\mathrm{c}}$ through the approximate solution of the Eliashberg equation, given the intra-molecular deformation potentials $g_{m}$. These, and the vibrational frequencies, were calculated using the quantum-chemical MNDO semi-empirical technique (15). This method has been successfully used previously on a wide variety of

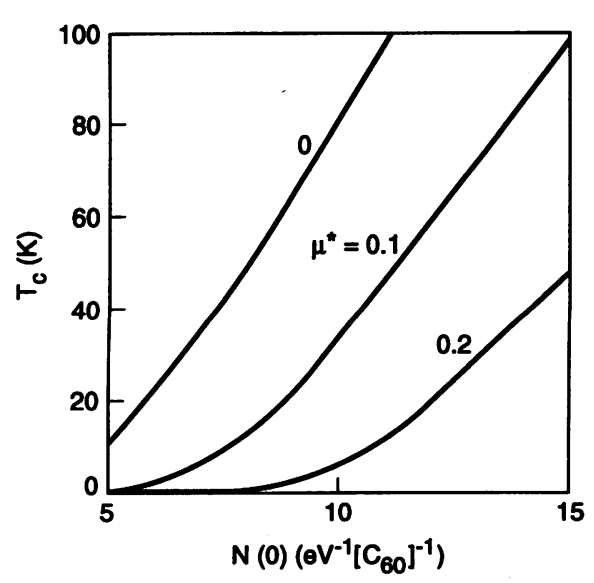

Fig. 4. $T_{c}$ (in kelvin) as a function of physically likely electronic densities of orbital states $N(0)$ and Coulomb pseudopotentials $\left(\mu^{*}\right)$.

Table 1. Experimental (20) and calculated frequencies $\left(\omega_{m}{ }^{\text {exp }}, \omega_{m}\right.$, in $\left.\mathrm{cm}^{-1}\right)$ and deformation potentials $\left(g_{m}\right.$, in $\left.\mathrm{eV} / \AA\right)$, and the corresponding coupling constants $\lambda_{m}$ for $N(0)=10$ per spin per $\mathrm{eV}$ per $\mathrm{C}_{60}$ molecule for the eight $H_{\mathrm{g}}$ modes of a $\mathrm{C}_{60}^{-}$molecule.

\begin{tabular}{lcccccccc}
\hline$\omega_{m}{ }^{\exp }$ & 273 & 437 & 710 & 744 & 1099 & 1250 & 1428 & 1575 \\
$\omega_{m}$ & 263 & 447 & 711 & 924 & 1260 & 1406 & 1596 & 1721 \\
$g_{m}$ & 0.1 & 0.1 & 0.2 & 0.0 & 0.6 & 0.2 & 1.8 & 1.2 \\
$\lambda_{m}$ & 0.03 & 0.01 & 0.01 & 0.0 & 0.06 & 0.0 & 0.34 & 0.11 \\
\hline
\end{tabular}

carbon compounds containing five- and sixmembered rings and is known to be reliable for structures, relative energies and vibrational frequencies (16). For example, the MNDO bond-lengths in $\mathrm{C}_{60} 1.47$ and 1.40 $\AA$ (17) are in excellent agreement with the best theoretical (18) and experimental (19) estimates. We evaluated the complete matrix of force constants and calculated the associated normal coordinates for all 174 vibrations in pure $\mathrm{C}_{60}$. In Table 1 the results for the eight Raman active $H_{g}$ mode frequencies are shown which have a mean deviation of only $10 \%$ from experiment $(20)$ (Table 1 ). We calculated the deformation potentials $g_{m}$ by a frozen-phonon technique. The energy of a $\mathrm{C}_{60}^{-}$molecule will depend on the amplitude of a frozen-in $Q_{m, 5}$ photon as $E=$ $-g_{m} Q_{m, 5}+\kappa_{m} Q_{m, 5}^{2} / 2\left(\kappa_{m}\right.$ is the spring constant in Eqs. 2 and 3). For each of the eight $H_{g}$ phonons we selected this component $\left(\sim 3 z^{2}-r^{2}\right)$, and we distorted the negatively charged $\mathrm{C}_{60}$ molecule along these normal coordinates. The initial slope of the energy as a function of the distortion amplitude yields then the deformation potentials.

In Table 1 the results for the deformation potentials are summarized, together with the coupling constants for the individual modes $\lambda_{m}$, as calculated from Eqs. 7 and 9 with the observed phonon frequencies in $\mathrm{C}_{60}$ and the phonon renormalization calculated below. We find that the two highest modes near 1428 and $1575 \mathrm{~cm}^{-1}$ are the most strongly coupled. The reason is that these high-lying modes involve bondstretching, compared to the bond-bending characterizing the lower frequency modes. The former leads to the maximum change in energy for a given (normalized) distortion.

Assuming a square density of states and a bandwidth, which is $\sim 0.6 \mathrm{eV}$ according to band structure calculations, $N(0)$ would be $\sim 5$ orbital states per electron volts per $\mathrm{C}_{60}$ molecule. However, the band structure calculations (21) give figures for $N(0)$ which are more than twice as large and experimentally $N_{\mathrm{o}} \sim 10$ to $20\left[H_{\mathrm{c} 1}, H_{\mathrm{c} 2}\right.$, normal state susceptibility (22)]. From Table 1 we find that the overall $\lambda=\Sigma_{m} \lambda_{m}$ is in the range of $\sim 0.3$ to 0.9 , and together with the large range in frequency for which the interactions are attractive, high $T_{\mathrm{c}}$ 's are to be expected. The large electron-phonon cou- pling also leads to a strong decrease in the phonon frequencies and a corresponding increase in their linewidth $=\gamma_{m} \omega_{m}$. It is known that (24)

$$
\frac{\Delta \omega_{m}}{\omega_{m}} \simeq-\frac{\lambda_{m}}{5} ; \frac{\gamma_{m}}{\omega_{m}} \simeq \pi N(0)\left|\Delta \omega_{m}\right|
$$

So we predict a diminution of about $5 \%$ in the frequency of the high $H_{g}$ modes and a linewidth increase of order $300 \mathrm{~cm}^{-1}$, compared to pure $\mathrm{C}_{60}$.

We now present an approximate calculation of $T_{\mathrm{c}}$. For $\lambda<1$, McMillan (13) has given a very good (23) approximate solution to the Eliashberg equations (12)

$$
T_{\mathrm{c}}=\frac{\omega_{\mathrm{av}}}{1.2} \exp \left[-\frac{1.04(1+\lambda)}{\lambda-\mu^{*}(1+0.62 \lambda)}\right]
$$

Most of the coupling strength is in the two highest lying modes, so the usual average of the phonon frequencies (23) $\left(\omega_{\log }\right)$ is not appropriate. We find

$$
\omega_{a \nu}=\exp \left\{\sum_{m} \frac{\lambda_{m} \ln \left[\omega_{m}\left(1-\lambda_{m}\right)\right]}{\lambda}\right\}
$$

a better approximation. Eq. 11 includes the Coulomb pseudo-potential parameter $\mu^{*}$. Because the approximations such as those due to Migdal (25) do not work for electron-electron interactions, it is impossible to estimate $\mu^{*}$ (in contrast to $\lambda$ ). Traditionally $\mu^{*}$ is used as a fitting parameter in comparing $T_{c}, \Delta(0)$ and the tunneling spectra to theory. For instance, for $\mathrm{Pb} \mu^{*}=0.12$ (23). $\mu^{*}$ for a Fermi-level in a well-isolated band is smaller than the screened repulsion parameter $\mu$ by a factor $\left[1+\mu \ln \left(W / \omega_{\text {av }}\right)\right]$ (26), where $W$ is the smaller of $E_{\mathrm{F}}$ and $\omega_{\mathrm{p}}$, the plasma frequency. $E_{\mathrm{F}} / \omega_{\mathrm{av}}$ in the Fullerenes is smaller than, say in $\mathrm{Pb}$, by $\sim 10^{2}$. However, if one notes that the actual electronic structure of metallic $\mathrm{C}_{60}$ is a ladder of bands of width $\sim 1 \mathrm{eV}$, spread out over $20 \mathrm{eV}$, and separated from each other by energies also of order $\mathrm{leV}$, and considers the calculation of $\mu^{*}$ in this situation, one concludes that $\mu^{*}$ is close in value to that of a wide band metal.

We present in Fig. $4 T_{\mathrm{c}}$ versus $N(0)$ for various values of $\mu^{*}(27)$. $T_{c}$ has a particularly simple relation to $N(0)$ and therefore to the nearest-neighbor $\mathrm{C}_{60}$ distance $d$ in the Fullerenes, because the other factors are 
intramolecular and do not depend on $d$. Figure 4 shows that $T_{c} \sim N(0)$, in agreement with a recent compilation of lattice constants, calculated densities of states and $T_{\text {c }}$ 's (21). Given the physical fact we used that most of the coupling is intramolecular, our estimate of $\lambda$ from the $H_{g}$ modes should be as good as the determination of vibration frequencies, that is, good to about $10 \%$. One worry is that our calculation of $g_{m}$ is based on the deformations of a $\mathrm{C}_{60}^{-}$molecule, whereas the more appropriate calculation would have a neutralizing background. The Migdal approximation for determining $T_{\mathrm{c}}$ is only good in our case to $\left(\omega_{\mathrm{av}} / E_{\mathrm{F}}\right) \sim$ $1 / 5$.

For low density of states obtainable by small doping, we expect the Coulomb interactions to dominate. In that case the intramolecular Hund's rule coupling (owing to orbital degeneracy) plus the almost empty band usually favors ferromagnetism (28). This may be the simple reason for the recent observation of ferromagnetism in the compound $\mathrm{TDAE}_{1} \mathrm{C}_{60}(29)$.

Note added in proof: In recent Raman measurements Duclos et al. (30) find that the two highest frequency $H_{g}$ phonons, which we find couple most strongly to the electrons (Table 1), are clearly seen in $\mathrm{C}_{60}$ and $\mathrm{K}_{6} \mathrm{C}_{60}$ but disappear in the superconducting compound $\mathrm{K}_{3} \mathrm{C}_{60}$. This is consistent with our prediction based on Eq. 10 for their linewidth.

\section{REFERENCES AND NOTES}

1. A. F. Hebard et al., Nature 350, 600 (1991); M. J. Rosseinsky et al., Phys. Rev. Lett. 66, 2830 (1991).

2. R. C. Haddon, L. E. Brus, K. Raghavachari, Chem. Phys. Lett. 125, 459 (1986); M. Ozaki and A. Takahashi, ibid. 127, 242 (1986).

3. S. Satpathi, ibid. 130, 545 (1986).

4. S. Saito and A. Oshiyama, Phys. Rev. Lett. 66, 2637 (1991).

5. J. L. Martins, N. Trouillier, M. Schnabel, preprint (University of Minneapolis, Minnesota, 1991)

6. S. Barisic, J. Labbe, J. Friedel, Phys. Rev. Lett. 25, 419 (1970); C. M. Varma, E. I. Blount, P. Vashista W. Weber, Phys. Rev. B 19, 6130 (1979); C. M. Varma and W. Weber, ibid., p. 6142.

7. C.-T. Chen et al., Nature, in press; J. H. Weaver et al., Phys. Rev. Lett. 66, 1741 (1991); M. B. Jost et al., Phys. Rev. B, in press.

8. M. C. O'Brien, J. Phys. C: Solid State Phys. 4, 2524 (1971)

9. In fact, in strong coupling one should consider the true $n$-particle states instead of simple product states as assumed in the text. This leads to an increase of phonon phase space, which however affects the 2,3 and 4 particle states in similar ways. Note that in thi case, the low spin states would be considered, and Jahn-Teller interactions thus give rise to a negative Hund's rule coupling.

10. C. M. Varma, Phys. Rev. Lett. 61, 2713 (1988); R Micnas, J. Ranninger, S. Robaskiewicz, Rev. Mod. Phys. 62, 113 (1990)

11. F. C. Zhang, M. Ogata, T. M. Rice, preprint (Univer sity of Cincinnat, Cincinnati, OH, 1991). This pape discusses the possibility that the alkali-ion vibrations may lead to an intramolecular electron-electron attraction. We find this unlikely because they screen their repulsive interactions without screening the attractive interactions. Besides, such vibrations have a frequency of $\mathbf{O}\left(200 \mathrm{~cm}^{-1}\right)$, much smaller than the molecular vibrations we consider and thus are unlikely to be of importance in determining $T_{c}$

12. G. M. Eliashberg, Zh. Eksp. Teor. Fiz 38, 966 (1960); ibid. 39, 1437 (1960) Sov. Phys.JETP 11, 696 (1960); ibid. 12, 1000 (1961)

13. W. L. McMillan, Phys. Rev. 167, 331 (1968).

14. In the derivation of Eq. 9 we missed the factor $5 / 6$ which was kindly pointed out to us by $M$. Lannoo.

15. M. J. S. Dewar and W. Thiel, J. Am. Chem. Soc. 99 4899 (1977); ibid., p. 4907.

16. M. D. Newton and R. E. Stanton, ibid. 108, 2469 (1986).

17. R. E. Stanton and M. D. Newton, J. Phys. Chem. 92, 2141 (1988)

18. M. Haesen, J. Almlof, G. E. Scuseria, Chem. Phys. Lett. 181, 497 (1991); K. Raghavachari and C. M. Rohlfing, $J$. Phys. Chem., in press.

19. C. S. Yannoni et al., paper presented at the Materials Research Society Meeting, Boston, 1990.

20. D. S. Bethune, Chem. Phys. Lett. 179, 181 (1991).

21. R. M. Fleming, Nature 352, 787 (1991).

22. A. P. Ramirez, M. J. Rosseinsky, D. W. Murphy, R. C. Haddon, in preparation.

23. P. B. Allen and R. C. Dynes, Phys. Rev. B 12, 905 (1975).

24. C. O. Rodriguez et al., Phys. Rev. B 42, 2692 (1990)

25. A. B. Migdal, Zh. Eksperim. $i$ Teor. Fiz. 34, 1438 (1958); Sov. Phys.-JETP 7, 996 (1958).

26. P. Morel and P. W. Anderson, Phys. Rev. 125, 1263 (1962); P. W. Anderson, in preparation.

27. An independent calculation of the electron-phonon coupling in the Fullerenes has been carried out by M. Schluter et al. (in preparation) using a tightbinding method. Our results differ in some ways from theirs. They find the major contribution from the $-400 \mathrm{~cm}^{-1}$ and $-1600 \mathrm{~cm}^{-1} H_{g}$ phonons, where the latter contribution is about half that of the former. In their calculation of $T_{c}$ they use an expression averaging over all the phonon frequencies irrespective of their coupling constants.

28. We envisage a competition between Hund's rule couplings and phonon-induced attractive interactions. We note the interesting possibility discussed by $S$. Chakravarty and S. Kivelson (Europhys. Lett., in press) that in a Hubbard model for $\mathrm{C}_{60}$ clusters, the effective electron-electron interaction is repulsive for small $U$; that is, the Hund's rule is obeyed, but for larger $U$ it may change sign due to configuration interactions.

29. P. M. Allemand et al., Science 253, 301 (1991).

30. S. J. Duclos, R. C. Haddon, S. Glarum, A. F. Hebard, K. B. Lyons, in preparation.

31. We thank B. Batlogg, R. C. Haddon, S. J. Duclos, I I. Mazin, M. F. Needels, T. T. M. Palstra, A. P. Ramirez, and M. Schluter for helpful discussions. We particularly thank $M$. Lannoo for carefully going through the manuscript and pointing out several compensating numerical errors. J.Z. acknowledges financial support by the Foundation of Fundamenta Research on Matter (FOM), which is sponsored by the Netherlands Organization for the Advancement of Pure Research (ZWO).

23 August 1991; accepted 3 October 1991

\title{
Long-Term History of Chesapeake Bay Anoxia
}

\author{
SHERri R. COOPER AND GRACE S. BRUSH
}

Stratigraphic records from four sediment cores collected along a transect across the Chesapeake Bay near the mouth of the Choptank River were used to reconstruct a 2000-year history of anoxia and eutrophication in the Chesapeake Bay. Variations in pollen, diatoms, concentration of organic carbon, nitrogen, sulfur, acid-soluble iron, and an estimate of the degree of pyritization of iron indicate that sedimentation rates, anoxic conditions and eutrophication have increased in the Chesapeake Bay since the time of European settlement.

S INCE THE OCCURRENCE OF ANOXIA was first reported in the Chesapeake Bay in the 1930's (1), the relative importance of climate versus eutrophication and other anthropogenic influences on the extent and duration of anoxic events has been debated (2-6). Large-scale monitoring of the chemical and physical properties of the Chesapeake Bay began as recently as 1984 (7). Information on the long-term history of anoxia in the Bay is available from stratigraphic records, accessed through paleoecological methods (8). In this report, we describe data from the stratigraphic records to evaluate conditions over time in the mesohaline (moderately brackish) section of the Chesapeake Bay.

Four sediment cores were collected in May 1985 along a transect across the Chesapeake Bay from the Choptank River to Plum Point, Maryland (Fig. 1). This region of the Bay is currently anoxic at least part of

Department of Geography and Environmental Engineer ing, Johns Hopkins University, Baltimore, MD 21218. each year Surface salinities for this area of the Bay average between 8 and 15 parts per thousand (ppt) in spring and fall, respectively; salinities in the bottom water are consistently higher and not usually less than $15 \mathrm{ppt}(9)$. The cores ranged in length from 114 to $160 \mathrm{~cm}$, and have a diameter of $5.7 \mathrm{~cm}$. The sediment in all cores was a relatively uniform mix of gray silt and clay, undisturbed by mixing or bioturbation. Each core was cut into $2-\mathrm{cm}$ intervals. The bottom sediments were dated by the carbon- 14 technique and the other samples were dated on the basis of pollen horizons and pollen concentration techniques (10). The agricultural pollen horizon was recognized by a sharp increase in concentration of ragweed pollen in relation to oak pollen and was dated as A.D. 1760 on the basis of historical records of land use in the area. Several controls were used to check the accuracy of both dating methods. For example, a sediment sample taken from above the agricultural pollen horizon in a nearby core could not be dated by radiocarbon 